\title{
Apprendre à programmer un robot humanoïde : impacts sur des élèves de l'adaptation scolaire
}

\section{Julien Bugmann \\ Université de Montréal \\ Thierry Karsenti \\ Université de Montréal}

Learning to program a humanoid robot:
impacts on special education
secondary-school students

doi:10.18162/fp. 2018.460

\section{ésumé}

L'enseignement du code prend de plus en plus d'importance dans la société, en particulier pour les jeunes qui seront les acteurs du monde technologique de demain. Cette recherche exploratoire visait à documenter, chez des élèves ayant des difficultés d'apprentissage, les impacts éducatifs liés à la programmation d'un robot humanoïde. Pour cela, nous avons utilisé une variété d'outils de collecte de données : observations vidéographiées, entrevues, etc. Sur le plan des résultats, en dehors du développement des compétences en codage des élèves, nous avons identifié différents impacts éducatifs, tels qu'une plus grande motivation pour l'école et une collaboration accrue entre les élèves.

\section{Mots-clés}

Codage, robot humanoïde, adaptation scolaire, apprentissage, école.

Abstract

Learning code is becoming an increasingly important aspect of modern society. In fact, computer programming has become an essential skill for all, especially children. The objective of this exploratory

study is to understand the educational impacts associated with "learning to code" a humanoid robot. To achieve this goal, we used a great variety of data collection methods: video observation, interviews, etc. Aside from an increase in coding skills, several educational outcomes were observed in students, including increased motivation with respect to school and school-related activities; increased collaboration and teamwork; and so on.

\section{Keywords}

Coding, humanoid robot, special needs, learning, school.

\section{Introduction}

Les parents attendent de l'école qu'elle permette à leurs enfants d'apprendre à compter, à lire, à parler d'autres langues et surtout qu'elle les amène vers une insertion sociale et professionnelle facilitée. Pourtant, on remarque que dans certains pays encore, les élèves sortent du système scolaire sans avoir acquis certaines de ces compétences qui, au vu des avancées technologiques actuelles, pourraient s'avérer importantes dans le monde de demain. Aussi, en plus du fait qu'il comporte une multitude d'impacts éducatifs (Smith, Sutcliffe et Sandvik, 2014), cet apprentissage du code est devenu obligatoire dans plusieurs pays tels que les États-Unis, la Grande-Bretagne, la France, la Suède et depuis peu, dans quelques provinces canadiennes.

Par ailleurs, plusieurs études et rapports (Duncan et Bell, 2015; Mubin, Stevens, Shahid, Mahmud et Dong, 2013) ont montré qu'apprendre à coder, y compris avec des robots, était de plus en plus important pour ces élèves, afin de leur permettre de comprendre le monde technologique qui les entoure et de mieux se préparer à y évoluer. Il s'agit donc là d'une compétence forte à acquérir par les jeunes (OECD, 2015). De façon globale, nous avons constaté qu'une partie des recherches qui traitent de l'apprentissage du code ne portent pas sur les élèves qui ont des difficultés d'apprentissage. Et encore bien moins d'études ont quant à elles évalué les impacts éducatifs de l'apprentissage du code sur ce public quand un robot est utilisé. Enfin, très peu ont porté sur l'apprentissage du code où un robot humanoïde sert d'outil à cet apprentissage, à l'exception de recherches portant sur des étudiants en école d'informatique, par exemple celle de Nijimbere, Boulc'H, Haspekian et Baron (2013). Ainsi, nous présentons dans cet article les résultats d'une recherche exploratoire revêtant une triple originalité par rapport aux autres 
travaux de recherche sur cette question. En effet, notre recherche porte sur l'apprentissage du code, par des élèves qui ont des difficultés d'apprentissage, dans un contexte très particulier où le code est appris en programmant un robot humanoïde qui parle, écoute, comprend, se déplace, etc. Dans le cadre de cette étude, les principaux objectifs étaient de mettre en évidence les avantages et défis liés à l'apprentissage du code par la programmation d'un robot humanoïde par des élèves ayant des difficultés d'apprentissage. Par ailleurs, l'intérêt de ce projet est majeur dans la mesure où il a été mis en évidence, comme nous l'avons dit, qu'apprendre à coder était particulièrement important pour le monde de demain, qu'apprendre le code via des robots pouvait s'avérer motivant et bénéfique aux apprenants et, surtout, qu'une telle initiative n'avait encore jamais été réalisée.

\section{Apprendre à programmer, pourquoi et comment?}

Avant tout, il nous apparaît comme important que de dissiper, d'emblée, un possible malentendu linguistique. En effet, nous avons conscience que certains chercheurs francophones insistent sur la différence entre les termes "programmation » et « code », où la programmation s'exprimerait par le code, qui est un ensemble d'instructions écrites en langage informatique, et qui permet de communiquer avec diverses technologies. Cependant, le terme de "codage " (ou coding en anglais) prend le même sens que la «programmation » comme le confirme l'Office québécois de la langue française (OQLF, 2001. Nous parlerons donc simplement de « codage ». Coder, c'est dire à un ordinateur, à un téléphone intelligent, à une application ou à un site internet ce qui doit être fait, à un moment donné, en réponse à telle ou telle action d'un utilisateur. Lorsque l'on code, on dirige le comportement de l'outil utilisé. Aussi, alors que les nouvelles technologies sont omniprésentes, il apparaît comme primordial de comprendre comment elles fonctionnent et pourquoi se produit telle ou telle réaction. En effet, le code est partout. Par exemple, lorsque l'on utilise son téléphone intelligent, un ordinateur, une tablette, un réseau social comme Facebook, ou encore une application aussi banale qu'un logiciel de traitement de texte, on génère un code qui n'est pas visible. Comprendre, ne serait-ce qu'en partie, ce que fait ce code, pourrait permettre aux utilisateurs de mieux comprendre le fonctionnement de l'outil et donc de mieux maîtriser ces multiples technologies qui nous entourent aujourd'hui. Comprendre le codage, c'est donc pouvoir anticiper certaines situations et se prémunir contre des risques. Alors que les métiers de demain risquent d'être fortement inspirés par le numérique, il est capital pour le devenir des jeunes et des moins jeunes qu'ils comprennent ce que l'on appelle la logique informatique.

C'est entre autres pourquoi l'apprentissage du code est aujourd'hui entré dans les salles de classe. Comme nous allons le constater, il n'est pas si difficile que cela de créer du code, que l'on soit petit ou grand, et que l'on ait des difficultés d'apprentissage ou non. Initiée par Seymour Papert (1981) il y a plusieurs dizaines d'années, cette éducation à la logique informatique, que l'on appelle souvent la «pensée informatique » (ou «computational thinking» en anglais) (Wing, 2006), se développe aujourd'hui de plus en plus grâce à des logiciels tels que Scratch ou Scratch Jr, et entre dans les salles de classe bien plus facilement qu'auparavant. Selon Wing (2006), cette pensée informatique est un «ensemble d'attitudes et d'acquis universellement applicables que tous, et pas seulement les informaticiens, devraient apprendre et maîtriser ». 
Les travaux de Papert reposaient sur un apprentissage de la logique informatique grâce à une petite tortue, appelée "LOGO », et que l'utilisateur allait apprendre à déplacer en la programmant. Le déplacement de cette tortue donnait alors lieu à un tracé sur l'écran qui permettait à l'apprenant de se repérer et de l'amener, d'une manière ludique, à la réalisation de visuels qui s'avéraient, pour certains, fort créatifs. Aujourd'hui, des logiciels tels que Scratch utilisent cette même logique d'apprentissage, mais avec davantage de réalisme.

\section{Impacts de l'apprentissage de la programmation sur les élèves}

Ces logiciels d'apprentissage de la programmation ont fait l'objet de nombreuses recherches sur leurs potentiels effets sur les utilisateurs (voir par exemple Sáez-López, Román-González et Vázquez-Cano, 2016; Ruf, Mühling et Hubwieser, 2014). Il a par exemple été mis en évidence que les utilisateurs de logiciels d'apprentissage de la programmation tels que Scratch comprenaient davantage les concepts et la logique de programmation après leur utilisation (Sáez-López, Román-González et Vázquez-Cano, 2016), mais qu'ils induisaient également une forte motivation dans la tâche, de l'engagement et du plaisir pour les élèves. Cela a même permis à ces derniers de créer du contenu lié à l'art et à l'histoire.

Par conséquent, certains pays ont mis en place "l'apprentissage du code » et l'ont même rendu obligatoire pour les élèves. Au Canada, où c'est le cas en Colombie-Britannique et en Nouvelle-Écosse, « coder à l'école » fait partie d'une plus vaste stratégie destinée à mieux préparer les jeunes pour l'avenir. Apprendre à coder revêt donc un intérêt particulier pour ces élèves (Falloon, 2016) qui vont pouvoir mieux comprendre le monde dans lequel ils vivent, mieux anticiper le futur et ce à quoi ils seront exposés afin, notamment, de mieux se préparer aux métiers de demain. Il s'agit donc d'une compétence forte pour les jeunes (OECD, 2015) d'autant plus que certaines recherches ont montré les avantages inhérents à l'apprentissage du code en éducation (Moreno León, Robles et Román-González, 2016). Parmi les principaux, on note, par exemple, des bénéfices en mathématiques (résolution de problèmes, attitudes face aux mathématiques, sentiment de compétence, etc.), de même que de meilleures habiletés en résolution de problèmes.

\section{Robotique pédagogique et robots humanoïdes pour apprendre}

Mais apprendre à coder, ce n'est pas seulement concevoir une histoire ou un jeu vidéo sur un écran d'ordinateur ou une tablette. Apprendre à coder, ça peut également être programmer un robot. Il est ainsi possible de programmer des robots de manière très simple, intuitive et pédagogique. C'est par exemple le cas du robot Dash qui peut être programmé par des élèves grâce à des applications intuitives et gratuites. Les élèves pourront ainsi, par exemple, faire effectuer au robot un parcours imaginé par l'enseignant en évitant des obstacles, en respectant certaines indications, etc. Et aujourd'hui, au même titre que Dash, de nombreux robots viennent soutenir ces enseignements et donner davantage de réalisme à l'apprentissage du code. On parle ainsi de plus en plus des robots Bee-Bot, des Lego Mindstorms, de Lego WeDo 2.0, de drones, de Sphero, de Pro-Bot ou encore d'Ozobot...

Certaines applications ont ainsi été proposées pour apprendre le code tout en contrôlant en même temps des robots. C'est ce que l'on appelle la robotique pédagogique, ou robotique éducationnelle (Gaudiello et Zibetti,2013), qui donne au robot une « nature d'objet réel et systémique ». Par la force des 
choses, ces robots ont progressivement investi les établissements scolaires et de nombreux chercheurs se sont penchés sur la question de leurs potentiels impacts sur les utilisateurs. Ce fut par exemple le cas pour Komis et Misirli (2013), qui ont étudié les processus de construction de programmes par des enfants en maternelle ayant des robots de type Bee-Bot, pour Kim et Lee (2016), qui ont analysé le recours aux robots et leurs effets positifs sur l'enseignement de la géométrie, mais aussi pour Kradolfer, Dubois, Riedo, Mondada et Fassa (2014) qui se sont intéressés aux effets du robot Thymio sur les enseignants. Cette dernière étude met en évidence l'apport de ces robots pour l'éducation d'élèves ayant des difficultés à suivre un programme d'études traditionnel. Ces auteurs avancent également que les enseignants souffrent d'un manque d'encadrement et de formation institutionnelle quant à l'usage des robots en éducation.

Toh, Causo, Tzuo, Chen et Yeo (2016) ont quant à eux montré, par leur revue de la littérature sur la question de l'usage des robots pour la petite enfance, que les bénéfices de tels outils pouvaient concerner quatre catégories majeures : les compétences cognitives, conceptuelles, linguistiques et sociales. Les auteurs mettent également en évidence le fait que ces robots permettent un développement de la compréhension des processus scientifiques, des concepts mathématiques, mais aussi de l'intérêt pour l'ingénierie.

On constate ainsi que le recours à des robots peut s'avérer efficace en termes d'apprentissage, ce que confirment Kazakoff, Sullivan et Bers (2013), et qu'ils permettent de développer une attitude positive envers la programmation, grâce par exemple à des outils tels que le site internet code.org (Kalelioğlu, 2015) qui, lui aussi, amène les enfants à programmer dans diverses situations. S'inscrivant dans une approche constructiviste de l'apprentissage (Komis et Misirli, 2013), la robotique pédagogique se situe "au carrefour de deux approches pédagogiques très fertiles au plan cognitif : les activités de manipulation et de construction des objets tangibles [...] et les micromondes programmables » (Komis et Misirli, 2013, p. 1). Aussi, dans un monde où, de plus en plus, les robots à forme humanoïde investissent les lieux publics (grandes surfaces, conférences, etc.), mais aussi privés, il nous est apparu comme indispensable que de proposer un rapprochement entre ces nouvelles technologies et les jeunes utilisateurs.

\section{Des robots humanoïdes rarement utilisés en classe de primaire à des fins d'apprentissage du code}

$\mathrm{Au}$-delà des "petits robots ", qui reprennent pour la plupart les fonctions propres aux voitures télécommandées (avance, tourne à droite, tourne à gauche, recule, etc.), quelques recherches ont eu recours à des robots plus évolués : on les qualifie de robots sociaux ou humanoïdes (Shiomi, Kanda, Howley, Hayashi et Hagita, 2015). Les robots humanoïdes ressemblent à un être humain : ils ont une tête, deux bras, deux jambes et se tiennent debout. Leur visage ressemble aussi, pour certains, à celui d'un être humain, avec des yeux et une bouche. Même leur « voix » peut être adaptée et modulée.

Mais pourquoi des robots humanoïdes sont-ils d'intérêt pour l'éducation? Premièrement, et comme nous l'avons évoqué en introduction à cet article, parce que ces innovations technologiques commencent à être de plus en plus présentes dans la société d'aujourd'hui et qu'elles risquent encore de modeler le monde de demain. Deuxièmement, parce que les premières recherches menées montrent que cet 
outil est susceptible d'avoir de nombreux effets positifs sur les jeunes utilisateurs, en ce qui concerne le développement de compétences techniques et d'habiletés sociales. Il s'agit d'ailleurs là de deux pans d'une insertion plus aisée pour ces enfants dans la société de demain. Enfin, plusieurs chercheurs ont utilisé le robot humanoïde NAO à des fins éducatives, en particulier à destination d'un public présentant des troubles du spectre autistique, comme nous allons le voir ci-après. Cependant, tous ces projets ayant recours au robot humanoïde concernent les interactions entre le robot et les apprenants (Shamsuddin et al., 2012) et non pas la programmation du robot par des élèves.

C'est le cas notamment auprès des élèves atteints de troubles du spectre de l'autisme (TSA). En effet, le fait que les robots humanoïdes ressemblent à un homme, mais ne possèdent pas les mêmes caractéristiques, telles que l'empathie, par exemple, en font d'excellents alliés de l'éducation auprès de ce public. Les TSA sont caractérisés par des altérations qualitatives dans les relations sociales, mais aussi dans la communication verbale et non verbale (Caudrelier et Foerster, 2015; Centelles, Assaiante, Etchegoyhen, Bouvard et Schmitz, 2012). Ainsi, l'enfant autiste aura davantage de difficultés à interagir, va préférer les jeux répétitifs et va être sujet à des troubles de la communication et à un déficit d'intérêt pour les autres personnes (Caudrelier et Foerster, 2015). Selon Caudrelier et Foerster (2015), le robot peut se substituer à l'éducateur pour enseigner des compétences à un enfant atteint de troubles autistiques, et notamment l'amener vers une meilleure conscience de son corps ou dans le développement du toucher, comme ce fut le cas dans les travaux de Robins, Amirabdollahian, Ji et Dautenhahn (2010). Caudrelier et Foerster (2015) parlent d'un apport «crucial» du robot dans les thérapies de l'autisme et particulièrement dans le «développement cognitif de l'individu ». D'autres recherches se sont intéressées à l'apport des robots sur ces enfants, par exemple Fridin (2014) qui a utilisé un robot interactif comme assistant de l'enseignant en racontant des histoires préenregistrées aux élèves en éducation préscolaire. Les résultats de cette étude montrent que les enfants ont aimé interagir avec le robot et qu'il s'est avéré être un excellent allié de l'enseignant. Les travaux de Kim et al. (2013) confirment cet impact positif du robot social sur les enfants atteints de troubles du spectre de l'autisme. Les auteurs ont ainsi démontré que l'utilisation d'un robot social, en tant que partenaire d'interaction, augmentait les interactions sociales de l'enfant atteint d'un trouble autistique avec l'adulte, et ce, davantage qu'un partenaire humain ou un jeu vidéo.

On relève donc de multiples usages des robots humanoïdes en éducation, mais rarement pour enseigner la programmation, et encore moins, pour l'enseigner à des élèves de primaire issus de l'enseignement adapté. C'est en cela que notre posture apparaît comme tout à fait originale. En effet, au vu de ces différents éléments, notre objectif de recherche est de tenter de mettre en évidence les avantages et défis liés à la mise en place d'un dispositif d'apprentissage de la programmation à l'aide d'un robot humanoïde en contexte d'enseignement adapté.

Plus précisément, nous nous sommes intéressés au développement de compétences en programmation des élèves et au développement de leur motivation lors de cette activité. Pour ce faire, nous avons opté pour une recherche-action avec une méthodologie de nature essentiellement qualitative qui respecte, par le fait même, la réalité de ces élèves. 


\section{Méthodologie}

Les recherches effectuées auprès d'élèves ayant des difficultés d'apprentissage comportent leur lot de défis méthodologiques et le recours à une méthode d'analyse qualitative nous est apparu comme une méthode particulièrement adaptée à notre démarche (Trudel, Simard et Vonarx, 2006). En effet, les méthodes de recherche plus traditionnelles en sciences humaines (utilisation de questionnaires, par exemple) ne sont pas toujours de mise, en raison particulièrement des difficultés que ces élèves rencontrent lorsqu'il s'agit de remplir ce type d'outil de collecte des données.

\section{Participants}

L'établissement dans lequel nous avons mené notre recherche se trouve au Québec (Canada). Il s'agit d'un établissement en adaptation scolaire qui propose une formule alternative et adaptée permettant à des élèves ayant des difficultés d'apprentissage d'obtenir un diplôme de qualifications. Ces établissements amènent les élèves vers davantage d'autonomie et aident les jeunes à s'inscrire dans une démarche de citoyens engagés et de travailleurs productifs. Les élèves de cet établissement ont la particularité d'être très réfractaires à la forme scolaire et au monde de l'école. C'est ces différents éléments qui ont motivé notre choix dans le public visé par cette recherche. En effet, si on laisse ces jeunes sans un dispositif adéquat, le risque est grand de les voir décrocher encore et, par la même occasion, de voir s'accroître la fracture numérique. Ces publics dits "fragiles" seraient effectivement plus susceptibles que les autres à se trouver distancés par les usages numériques dans la société et à en être progressivement exclus (Alberola, Croutte et Hoibian, 2016). Il s'agit donc d'un public d'élèves que l'on dit défavorisés et qui ont plus de difficultés que les autres à s'émanciper, à s'intégrer et à prendre une place valorisante au sein de la société. Nous avons ainsi veillé à mettre en place un dispositif adapté à ces élèves ayant des besoins particuliers en rendant «capacitante » pour tous la situation d'enseignement-apprentissage (Benoit et Feuilladieu, 2017).

Au final, notre recherche, menée entre septembre 2016 et juin 2017, a concerné 7 enseignants ainsi que 79 de leurs élèves (34 filles et 45 garçons). Ces derniers avaient tous des difficultés d'apprentissage et étaient âgés de 12 à 18 ans. 


\section{Outils de collecte de données}

Dans le cadre de cette étude, et en lien avec nos objectifs de recherche, nous avons utilisé cinq méthodes de collecte des données qui sont présentées dans le tableau 1 ci-dessous :

\section{Tableau 1}

Principales méthodes de collecte des données.

\begin{tabular}{|l|l|}
\hline \multicolumn{1}{|c|}{ Méthode de collecte des données } & \multicolumn{1}{c|}{ Fréquence } \\
\hline $\begin{array}{l}\text { Des observations filmées (12 séances de } 90 \text { minutes) } \\
\text { lors desquelles les élèves apprenaient à coder un robot } \\
\text { humanoïde NA0 }\end{array}$ & 12 séances de 90 minutes auprès de 79 élèves \\
\hline Des interviews de groupe avec les enseignants & 2 séances de 30 minutes auprès de 7 enseignants \\
\hline Des entretiens de groupe avec les élèves & 4 séances de 25 minutes auprès de 79 élèves \\
\hline Des entretiens individuels avec les élèves & 4 séances de 5 minutes auprès de 79 élèves \\
\hline $\begin{array}{l}\text { L'état d'achèvement des niveaux de compétences liés au } \\
\text { codage du robot humanoïde }\end{array}$ & 79 documents relevés \\
\hline $\begin{array}{l}\text { Des analyses de traces (Larose et Jaillet, 2009) avec le relevé } \\
\text { des programmes réalisés par les élèves }\end{array}$ & $\begin{array}{l}\text { Enregistrement et captures d'écran des programmes créés } \\
\text { par les élèves avec le logiciel Choregraphe }\end{array}$ \\
\hline
\end{tabular}

\section{Méthode d'analyse des données}

Nous avons procédé à une analyse qualitative des réponses ouvertes aux entretiens, effectuée à l'aide du logiciel QDA Miner. Elle a consisté en une analyse de contenu (L'Écuyer, 1990; Miles et Huberman, 2003) dont le codage a été élaboré à partir des réponses des participants et en lien avec les objectifs de la recherche, soit l'apprentissage de la programmation, la motivation pour cet apprentissage et enfin le développement de la collaboration entre les apprenants. La base théorique de départ pour ce codage a été conçue à partir d'une revue de la littérature sur les avantages des robots en éducation.

\section{Forces et limites méthodologiques}

Dans notre étude, l'une des principales forces réside dans la méthodologie de recherche particulière employée. En effet, en procédant à une recherche dans laquelle tous les membres de l'établissement ont été impliqués, ainsi qu'à des entrevues individuelles et de groupe et à des observations filmées, cela est, en soi, un avantage pour enrichir et trianguler les résultats obtenus. Par ailleurs, et comme nous l'avons d'ores et déjà précisé, le fait de s'appuyer sur une méthode qualitative ne peut que donner davantage de poids et d'intérêt à une telle recherche (Trudel et al., 2006). Cependant, il est à noter certaines limites qui accompagnent ces choix méthodologiques. En effet, le travail sur la perception des participants reste une limite que nous avons tenté de pallier en croisant de nombreuses données (entretiens, enregistrements vidéo, performances des élèves, analyse des traces). Nous avons aussi, en permanence, confronté les réponses des participants afin de mettre en évidence toute divergence si nécessaire. 


\section{Dispositif mis en place}

Nous avons choisi d'utiliser le robot humanoïde NAO, créé par la société Aldebaran Robotics (désormais appelée SoftBank Robotics). Ce robot, qui mesure 58 centimètres et pèse 4,8 kilogrammes, est composé de deux caméras, de différents capteurs, mais aussi de microphones. Il peut entendre ce qu'il se passe autour de lui, voir et reconnaître les personnes et les objets devant lui. Il lui est donc possible d'interagir avec des humains. Presque exclusivement présent dans le milieu universitaire, le robot NAO a la particularité d'être programmable par tout utilisateur, grâce à un logiciel appelé Choregraphe. Ce qui signifie que les enfants, eux aussi, seraient susceptibles de pouvoir le programmer. Encore faut-il les y amener. En effet, dans la littérature, il n'a jamais été fait mention d'apprenants, au niveau primaire ou secondaire, qui auraient eu à manipuler ce robot en le programmant. À l'aide du logiciel Choregraphe, les élèves peuvent pourtant programmer le robot, le faire parler, le faire bouger, etc. Du moins, c'est le postulat que nous avons formulé.

Nous souhaitions donc utiliser ce robot pour amener des élèves ayant des difficultés d'apprentissage à s'intéresser à l'informatique et à s'initier au codage, tout en s'amusant. Cette pratique est, comme nous l'avons précisé, relativement absente encore aujourd'hui et les usages réguliers de ce robot interviennent majoritairement lorsqu'il s'agit d'un public d'élèves passifs et non pas actifs (les enfants ne sont pas amenés à le programmer). C'est d'ailleurs, rappelons-le, ce qui a déjà été effectué auprès d'un public d'élèves autistes, pour lesquels des modules d'interaction ou de mouvements destinés à amener les enfants à interagir ont été mis en place (Karsenti, Bugmann et Frenette, 2017; Shamsuddin et al., 2012). Enfin, le robot NAO a aussi été utilisé auprès d'élèves ayant des difficultés en écriture, mais cela ne fut pas réalisé par l'apprentissage du code (Lemaignan et al., 2016).

\section{La création d'un dispositif ludique et éducatif}

Dans le cadre de cette recherche, et pour baliser l'usage de NAO en classe, nous avons procédé à la création d'un programme scolaire : Devenez le maître NAO (figure 1). Nous avons donc créé 10 niveaux, composés chacun de 3 étapes intermédiaires, à réaliser par les élèves. Ces niveaux permettaient de découvrir et de maîtriser progressivement la méthode de programmation du robot NAO. Ainsi, le premier niveau proposait uniquement aux élèves d'interagir avec le robot par la voix et la manipulation physique, et ceci en sollicitant les programmes installés dans le robot. Cela avait pour objectif de stimuler non seulement la pratique langagière des élèves à destination d'un outil numérique, mais aussi de les amener vers une compréhension de la façon dont entend et comprend un robot de ce type (voir figure 2). Il s'agissait pour nous d'une étape primordiale dans la compréhension du fonctionnement de l'informatique et de la programmation par les élèves. Les niveaux proposés étant ainsi composés de trois activités intermédiaires, l'enfant avait finalement 30 activités au total à réaliser pour atteindre le niveau le plus élevé et devenir ce que nous avions appelé, un « Maître NAO ». En articulant de manière cohérente les boîtes de commandes à paramétrer dans Choregraphe, les élèves pouvaient faire agir le robot NAO et découvrir ainsi les effets de leur programmation. Ce programme scolaire «Devenez le maître $\mathrm{NAO}$ » a par ailleurs été conçu pour permettre un apprentissage progressif des techniques de programmation (activation d'une commande au niveau 2, création d'une séquence de commandes au niveau 4 , programmation séquentielle au niveau 5 , etc.). 


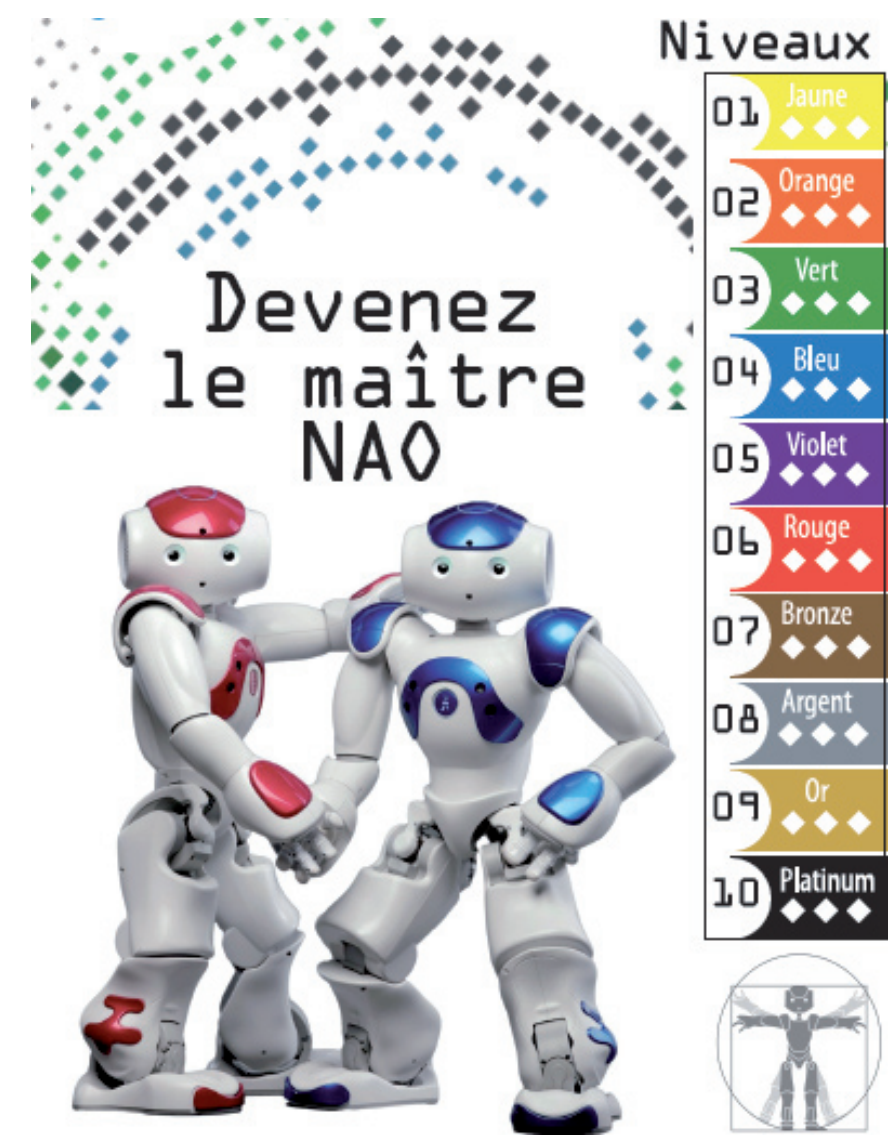

\section{Figure 1}

Affiche du programme scolaire « Devenez le maître NAO ».

Pour l'ensemble du logiciel Choregraphe, nous avons conçu trois guides afin d'accompagner les utilisateurs : un guide général de l'enseignant, un guide d'activités pour les élèves et un guide de correction pour les enseignants. Le guide général de l'enseignant intégrait toutes les informations nécessaires à la réalisation des différents niveaux ainsi que des fonctionnalités avancées, alors que le guide de l'élève était quant à lui davantage centré sur les niveaux à atteindre et proposait une aide concrète pour atteindre ces niveaux. Ainsi, l'élève pouvait se référer au guide pour accomplir telle ou telle activité et pouvait donc travailler en complète autonomie. Par ailleurs, nous avons conçu un guide de correction pour les enseignants afin qu'ils puissent très rapidement valider le niveau passé par l'apprenant. Dans ce dernier document, l'enseignant ne voyait que les boîtes de programmation à utiliser et l'ordre dans lequel elles devaient apparaître. Il pouvait ainsi valider rapidement chaque activité de l'élève.

D'un point de vue technique, nous avons rendu accessibles ces différents documents additionnels sur des tablettes tactiles afin que chaque élève puisse rapidement accéder à l'activité et aux consignes qui l'accompagne. 


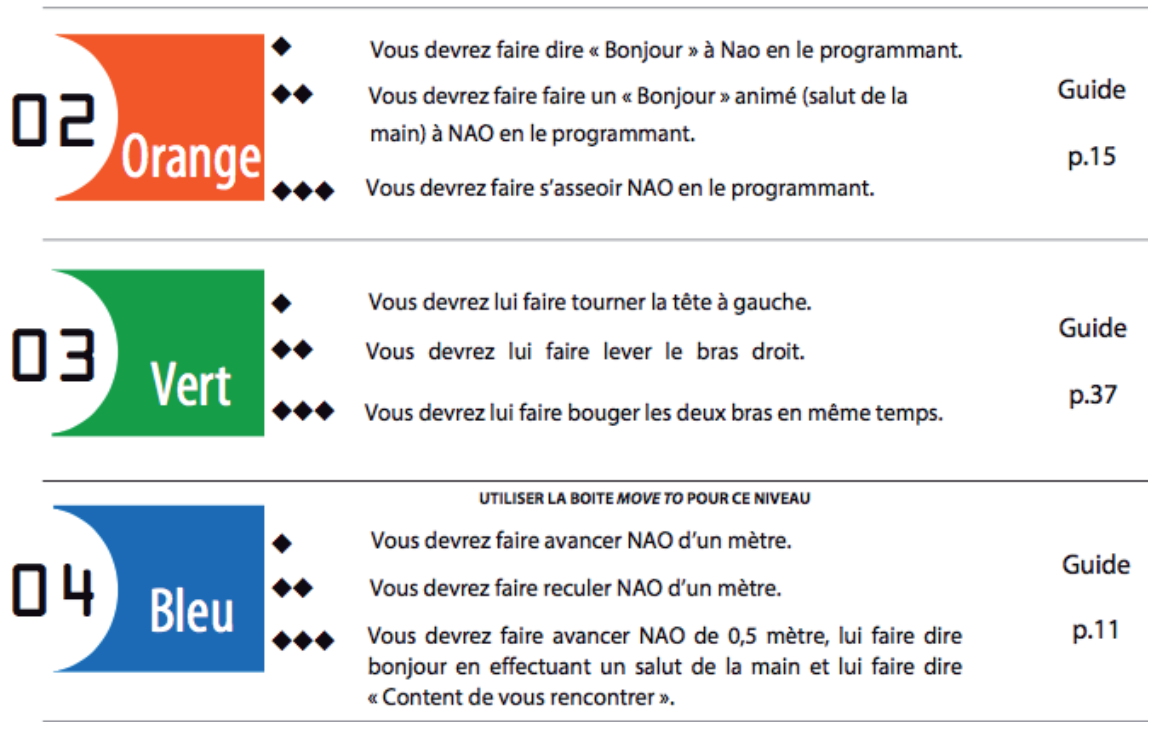

Figure 2

Exemples d'activités à réaliser en programmant le robot humanoïde NAO.

\section{Résultats}

Après exploitation et croisement des données collectées lors de cette recherche-action, et en lien avec nos objectifs de recherche, nous avons pu identifier une série d'avantages et d'inconvénients liés à l'apprentissage du code, par des élèves du secondaire en adaptation scolaire, à l'aide d'un robot de type humanoïde.

\section{Une forte motivation des élèves pour utiliser NAO}

Tout d'abord, les données vidéo recueillies ont mis en évidence le développement d'une forte motivation des élèves à se rendre à l'école et une ambiance de groupe très positive lors des séances de travail avec le robot NAO. Le fait de participer à une activité amenant, selon les élèves, du " fun ", a fortement stimulé la motivation des élèves, comme en témoignent les déclarations que nous avons recueillies lors des entrevues avec les élèves :

- «moi, j'ai aimé programmer NAO»

- "j'aime ça avec NAO, on peut faire plein de choses avec"

- «c'était le fun»

- "j’ai aimé le faire danser»

- "j'ai aimé le faire bouger"

- "c'est un atelier que j'ai beaucoup aimé»

- «j'ai pas mal tout aimé»

- «j'ai aimé le programmer pour qu’il danse » 
Certains des élèves ont même poursuivi des projets de programmation au-delà de nos visites et en dehors des heures de cours imposées. Les enseignants nous faisant ainsi parvenir des extraits vidéo des productions des élèves ou publiaient ces séquences sur les réseaux sociaux. Toujours grâce aux entrevues effectuées auprès des enseignants, nous avons appris que trois élèves de l'établissement ont effectué des formations à la programmation du robot NAO à 6 enseignants de l'établissement ainsi qu'à plusieurs dizaines d'enseignants du réseau lors d'une journée de formation aux usages de NAO. Le fait de recourir au robot humanoïde $\mathrm{NAO}$ a donc développé la motivation des élèves à se rendre en classe, que cela soit avant, pendant, ou après nos séances avec le robot.

\section{Un impact sur la collaboration entre les élèves}

Les données vidéo collectées ont également mis en évidence une collaboration accrue entre les élèves lors des séances de programmation (voir figure 3), mais aussi entre les élèves et les enseignants, et une plus grande entraide entre les élèves lors des séquences de debugging. Les élèves devaient ainsi trouver la solution à un problème rencontré dans la programmation et comprendre pourquoi telle ou telle programmation ne fonctionnait pas. Ils recommençaient alors leurs programmes jusqu'à ce que ces derniers soient fonctionnels afin de réussir les niveaux proposés. Cela va dans le sens des conclusions de Toh et al. (2016) qui parlaient d'un impact social fort du robot auprès des élèves. Le robot pourrait donc également servir à stimuler les activités sociales et donc collaboratives des jeunes utilisateurs, y compris ceux ayant davantage de difficultés que les autres.

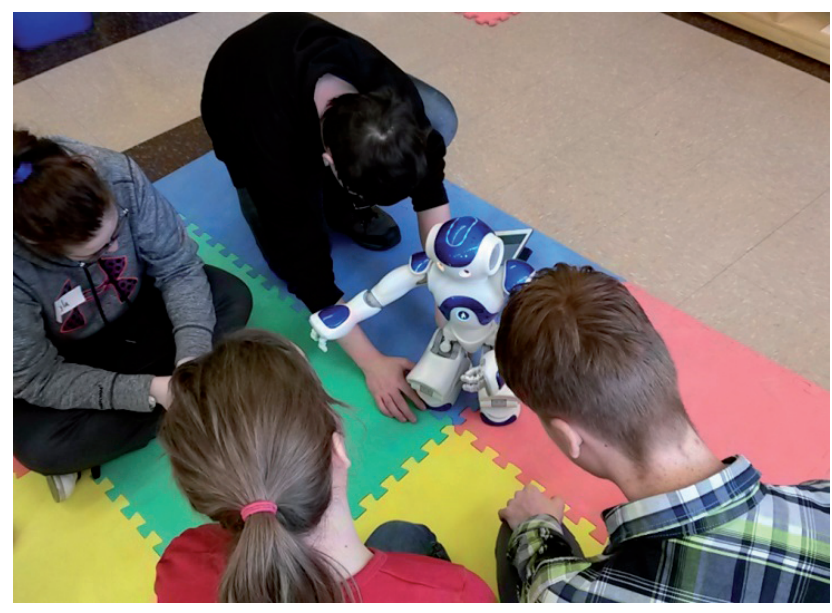

Figure 3

Des élèves manipulent le robot NAO.

Les données recueillies par le biais de nos entrevues avec les élèves, mais aussi par l'analyse d'un « livre des élèves finissants ", ont montré que ces élèves pouvaient travailler de manière autonome, y compris avec un robot si complexe. Dans le livre des finissants de l'année, un commentaire d'un camarade de classe concernant son ami résume bien la relation entre le robot NAO et les élèves :

"À l'école, il a un petit frère nommé NAO, il s’occupe de lui quand il ne veut plus travailler dans ses cahiers et il fait des programmations extraordinaires ». 


\section{Une stimulation de la créativité des élèves}

Aussi, la programmation du robot NAO, et notamment le niveau 10 proposé, a permis aux élèves de développer leur créativité, tout particulièrement quand il s'agissait de créer des comportements non explorés avec le robot. Les données vidéo ont ainsi montré que certains élèves lui ont fait faire un dab et que d'autres lui ont fait faire une chorégraphie avec une musique de film. Ces éléments ont été relevés dans les analyses de traces du logiciel, tels que les programmes créés par les élèves (voir figure 4 et figure 5), mais aussi dans les enregistrements vidéo analysés.

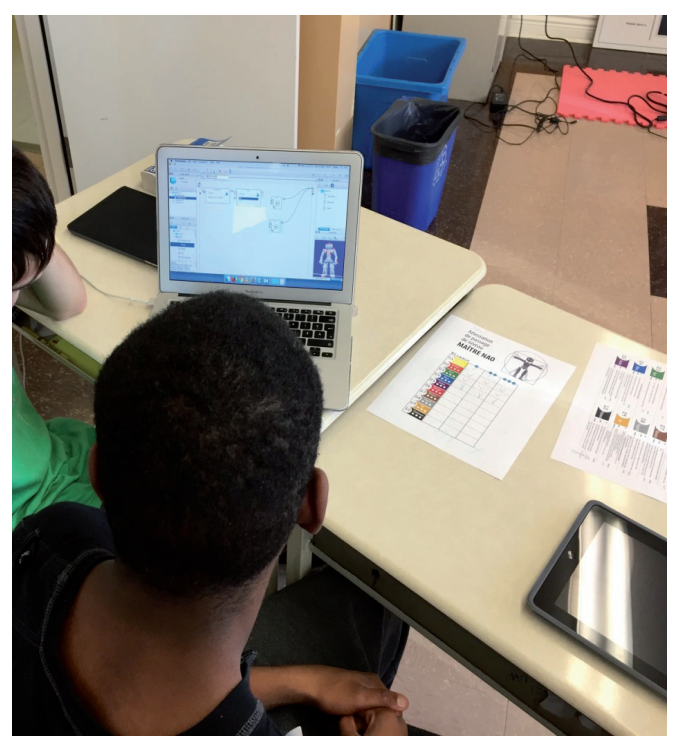

\section{Figure 4}

Utilisation du logiciel Choregraphe par des élèves.

Les données recueillies lors des entrevues individuelles et de groupe ont également mis en évidence le développement d'un sentiment de compétence à l'école plus élevé et, plus généralement, une meilleure estime de soi à l'école pour ces élèves : «je me sens beaucoup plus confiant à l'école ». D'autant que les élèves ont le sentiment de développer de nouvelles compétences : «ce qui est le fun c'est d'apprendre à le programmer, on apprend d'autres choses [...] mes connaissances s'agrandissent ». Nous avons ainsi pu constater un développement des compétences des élèves en codage et en pensée informatique par la réalisation des différents programmes pour contrôler le robot, mais aussi dans l'analyse de la progression des niveaux atteints par les élèves à l'issue des séances. En effet, ces données montrent que plus de $50 \%$ des élèves sont parvenus à atteindre le niveau 10, et donc à finaliser le programme " Maître NAO ». 


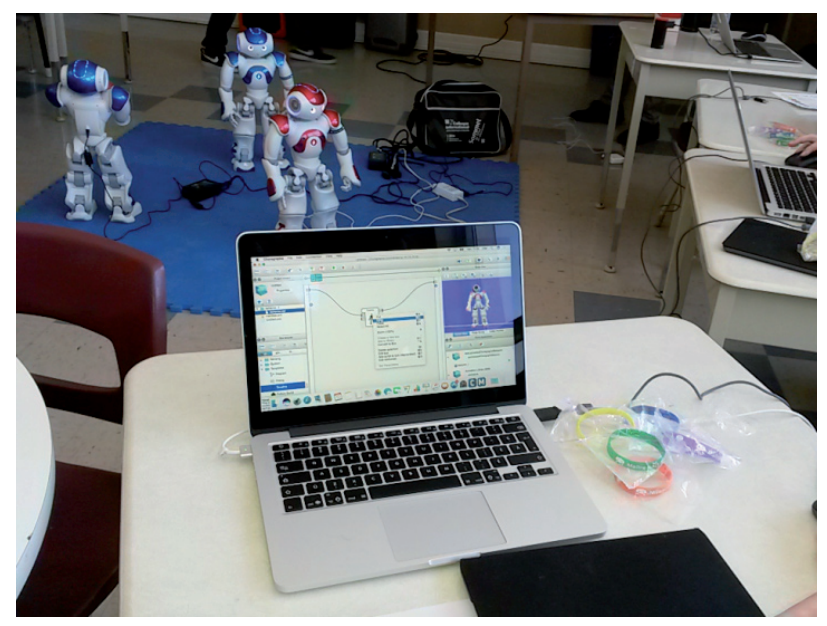

Figure 5

Un élève programme $\mathrm{NAO}$ avec le logiciel Choregraphe.

\section{D’autres apprentissages plus inattendus}

Enfin, les données recueillies lors des entrevues ont montré que ce dispositif avait permis aux élèves de stimuler leurs compétences en communication écrite et orale, notamment lorsqu'il s'agissait de faire parler le robot (rédaction de texte, adaptation du vocabulaire, respect de la ponctuation, etc.). Les élèves disent à ce sujet :

- "j’aime, car quand tu parles, il te répond à ce que tu parles»

- "j’ai aimé le faire discuter avec nous»

Nous aurions finalement pu signaler d'autres bénéfices pour les élèves, tels que l'apprentissage d'une langue seconde, à savoir, l'anglais, par exemple, dans la mesure où le logiciel de programmation est intégralement en anglais, mais aussi la recherche d'information nécessaire pour certains niveaux, mais ces éléments feront l'objet de publications futures.

\section{Certains défis subsistent}

Certaines contraintes sont toutefois à signaler, comme la complexité du logiciel de programmation utilisé, Choregraphe, ce que confirment les commentaires de certains élèves recueillis lors des entrevues de groupe notamment : «il y a des petites affaires qui étaient difficiles » ou « c'est dur » (de le programmer). Les analyses vidéo ont aussi montré que les élèves avaient parfois du mal à effectuer les programmes demandés (notamment pour les niveaux les plus élevés).

Autre difficulté, davantage technique, à savoir la connexion WiFi à obtenir avec le robot. En effet, pour se connecter au robot, et donc pouvoir l'utiliser avec le logiciel de programmation, il était indispensable d'être lié au même réseau que lui. Dans une école, cela représente un défi majeur, en raison des nombreuses restrictions et mesures de sécurité existantes. Après avoir trouvé la solution, en associant chaque robot à une borne WiFi mobile unique que nous amenions en classe, nous avons 
pu mettre en place les séances d'une manière tout à fait fluide et stable. Aussi, la manipulation du robot par les élèves devait demeurer douce et bienveillante, ce qui amène, par la même occasion, à une responsabilisation des élèves codeurs lorsqu'ils manipulent un tel outil. En effet, ce robot a un certain prix, cela peut représenter une difficulté pour un établissement d'enseignement. Enfin, l'ajustement des tâches de programmation à réaliser a également nécessité un certain temps d'adaptation, et un certain nombre d'essais-erreurs a été indispensable pour finaliser le dispositif dans la mesure où celui-ci n'avait jamais été éprouvé par le passé.

\section{Discussion et conclusion}

Finalement, les résultats de cette recherche ont mis en évidence de nombreux avantages éducatifs liés à l'usage d'un robot humanoïde en contexte éducatif, et pas uniquement en ce qui concerne l'apprentissage "pur» de la programmation. En effet, nous avons relevé une très forte motivation des apprenants et de leurs enseignants pour ce projet. Aussi, et malgré les défis rencontrés dans la mise en place de cette recherche-action, nous avons pu assister dans les salles de classe au développement de compétences en programmation par les élèves qui ont, pour certains, en deux séances de 3 heures seulement, pu atteindre le dixième niveau proposé dans notre dispositif. Ce résultat prend encore plus d'ampleur lorsque l'on sait que ce type d'usage n'avait été fait qu'auprès d'élèves du secondaire (Nijimbere et al., 2013) et certainement pas auprès d'élèves du primaire ayant des difficultés d'apprentissage. Tous ces éléments démontrent que, même si l'on présente ces élèves comme ayant des difficultés d'apprentissage, il n'en reste pas moins qu'ils peuvent être performants, tout particulièrement dans le domaine de la programmation informatique qu'ils ne connaissaient pas et qui reste inexploré pour beaucoup de personnes.

Autre avantage majeur constaté, c'est la fréquence élevée des interactions entre les participants lors des séances de programmation du robot NAO. En effet, les élèves étaient très enjoués et collaboraient bien plus que lors de leurs enseignements traditionnels. Rappelons à cet effet que ces élèves n'apprécient guère l'école et que notre recherche a montré que le fait d'utiliser et de programmer un tel robot humanoïde les a stimulés et a généré chez eux un fort intérêt à se rendre dans leur établissement scolaire. Par ailleurs, certains milieux ont pu poursuivre leur intégration de la robotique avec l'accessibilité permanente à Choregraphe et même dans certains cas la possibilité de prolonger les usages une fois notre équipe partie, avec un robot humanoïde qu'ils ont décidé d'acquérir, malgré le coût important que cela représente.

On constate donc qu'il y a eu un effet particulièrement positif du recours au robot humanoïde NAO pour l'apprentissage de la programmation informatique en contexte d'enseignement adapté au niveau secondaire. Alors même que l'apprentissage du code est de plus en plus valorisé et encouragé, tant par les décideurs politiques que par les chercheurs, nous avons pu relever à quel point un tel dispositif était en mesure d'amener de multiples opportunités pour tous les élèves. Ainsi, bien plus qu'un simple jouet, le robot humanoïde NAO pourrait s'avérer être un allié pour l'éducation des jeunes par le développement

de compétences en codage, mais aussi de diverses compétences et connaissances qui appartiennent au domaine scolaire ainsi qu'à la vie professionnelle et sociale. 
Pourtant, il est à noter que très peu d'élèves de nos écoles sont, à ce jour, exposés à cet automate du futur, alors même qu'il pourrait être intéressant de chercher à initier tous les élèves, futurs membres de la société, à cette nouvelle technologie qui risque d'être encore plus présente dans la société de demain (Hood, Lemaignan et Dillenbourg, 2015). En effet, ces élèves qui ne suivent plus ou ne peuvent plus suivre le réseau traditionnel d'éducation scolaire, qui ont besoin de ressources différenciées pour apprendre, et qui ont des difficultés d'insertion dans la société, doivent être, encore plus que les autres, amenés vers une meilleure utilisation et compréhension des outils numériques existants.

Il est enfin à signaler que ce projet n'a pas pris fin à l'issue des séances. Nous avons pu constater, grâce à des échanges de vidéo de la part des enseignants et du directeur de l'école, un prolongement de notre projet avec la création de nouveaux programmes, pour certains particulièrement innovants et complexes. Il serait d'ailleurs particulièrement intéressant de penser à une intégration généralisée et réaliste de ce type d'outil pour les milieux. Pour cela, il faudrait également que les enseignants bénéficient de formations adéquates, eux qui, avec le robot Thymio, souhaitaient déjà être accompagnés (Kradolfer et al., 2014).

La formule reste donc à définir mais, à l'issue de notre projet, nous montrons que ce type de réalisation peut être efficace d'un point de vue pédagogique.Des propositions d'intégration en classe, mais également d'autres initiatives menées par les élèves avec ce robot feront l'objet de publications futures.

\section{Références}

Alberola, É., Croutte, P. et Hoibian, S. (2016). La « double peine » pour des publics fragilisés face au tout-numérique. Annales des Mines - Réalités industrielles, 2016(3), 32-36. Repéré à http://www.annales.org/ri/2016/ri-aout-2016/RI-aout-2016-Alberola-Croutte-Hoiban.pdf

Benoit, H. et Feuilladieu, S. (2017). De la typologie des outils numériques dans le champ des EIAH à leur opérationnalité inclusive. La nouvelle revue de l'adaptation et de la scolarisation, (78), 25-45. http://dx.doi.org/10.3917/nras.078.0025

Caudrelier, T. et Foerster, F. (2015). Contribution des robots sociaux aux thérapies des troubles du spectre autistique : une revue critique. Dans G. Bailly et S. Petsy (dir.), IC2A (p. 25-32). Repéré à https://hal.archives-ouvertes.fr/cel-01110281/file/TER2015.pdf

Centelles, L., Assaiante, C., Etchegoyhen, K., Bouvard, M. et Schmitz, C. (2012). Understanding social interaction in children with autism spectrum disorders: does whole-body motion mean anything to them?. L'Encéphale, 38(3), 232-240. http://dx.doi.org/10.1016/j.encep.2011.08.005

Duncan, C. et Bell, T. (2015). A pilot computer science and programming course for primary school students. Dans J. GalEzer, S. Sentance et J. Vahrenhold (dir.), Proceedings of the Workshop in Primary and Secondary Computing Education (p. 39-48). New York, NY : ACM. http://dx.doi.org/10.1145/2818314.2818328

Falloon, G. (2016). An analysis of young students' thinking when completing basic coding tasks using Scratch Jnr. on the iPad. Journal of Computer Assisted Learning, 32(6), 576-593. http://dx.doi.org/10.1111/jcal.12155

Fridin, M. (2014). Storytelling by a kindergarten social assistive robot: A tool for constructive learning in preschool education. Computers $\mathcal{E}^{2}$ Education, 70, 53-64. http://dx.doi.org/10.1016/j.compedu.2013.07.043

Gaudiello, I. et Zibetti, E. (2013). La robotique éducationnelle : état des lieux et perspectives. Psychologie française, 58(1), 17-40. http://dx.doi.org/10.1016/j.psfr.2012.09.006

Hood, D., Lemaignan, S. et Dillenbourg, P. (2015). When children teach a robot to write: An autonomous teachable humanoid which uses simulated handwriting. Dans J. A. Adams et W. Smart (dir.), Proceedings of the Tenth Annual ACM/IEEE International Conference on Human-Robot Interaction (p. 83-90). New York, NY : ACM. http://dx.doi.org/10.1145/2696454.2696479 
Kalelioğlu, F. (2015). A new way of teaching programming skills to K-12 students: Code.org. Computers in Human Behavior, 52, 200-210. http://dx.doi.org/10.1016/j.chb.2015.05.047

Karsenti, T., Bugmann, J. et Frenette, E. (2017). Un robot humanoïde pour aider les élèves ayant un trouble du spectre de l'autisme?. Vivre le primaire, 30(2), 34-37.

Kim, E. S., Berkovits, L. D., Bernier, E. P., Leyzberg, D., Shic, F., Paul, R. et Scassellati, B. (2013). Social robots as embedded reinforcers of social behavior in children with autism. Journal of Autism and Developmental Disorders, 43(5), 1038-1049. http://dx.doi.org/10.1007/s10803-012-1645-2

Kim, S. et Lee, C. (2016). Effects of robot for teaching geometry to fourth graders. International Journal of Innovation in Science and Mathematics Education (Formerly CAL-Laborate International), 24(2). Repéré à https://openjournals.library.sydney.edu.au/index.php/CAL/article/view/9048

Komis, V. et Misirli, A. (2013, octobre). Étude des processus de construction d'algorithmes et de programmes par les petits enfants à l'aide de jouets programmables. Communication présentée à Sciences et technologies de l'information et de la communication (STIC) en milieu éducatif. Repéré à https://edutice.archives-ouvertes.fr/edutice-00875628/document

Kradolfer, S., Dubois, S., Riedo, F., Mondada, F. et Fassa, F. (2014). A sociological contribution to understanding the use of robots in schools: The Thymio robot. Dans M. Beetz, B. Johnston et M.-A. Williams (dir.), Social Robotics (p. 217-228). Cham : Springer. http://dx.doi.org/10.1007/978-3-319-11973-1 22

Larose, F. et Jaillet, A. (dir.). (2009). Le numérique dans l'enseignement et la formation : analyses, traces et usages. Paris : L'Harmattan.

L'Écuyer, R. (1990). Méthodologie de l'analyse développementale de contenu: méthode GPS et concept de soi. Québec, QC : Presses de l'Université du Québec.

Lemaignan, S., Jacq, A., Hood, D., Garcia, F., Paiva, A. et Dillenbourg, P. (2016). Learning by teaching a robot: The case of handwriting. IEEE Robotics and Automation Magazine, 23(2), 56-66. http://dx.doi.org/10.1109/mra.2016.2546700

Miles, M. B. et Huberman, A. M. (2003). Analyse des données qualitatives. Louvain-La-Neuve : De Boeck Supérieur.

Moreno León, J., Robles, G. et Román-González, M. (2016). Code to learn: Where does it belong in the K-12 curriculum?. Journal of Information Technology Education: Research, 15, 283-303. http://dx.doi.org/10.28945/3521

Mubin, O., Stevens, C. J., Shahid, S., Mahmud, A. A. et Dong, J.-J. (2013). A review of the applicability of robots in education. Technology for Education and Learning, 1(1). http://dx.doi.org/10.2316/Journal.209.2013.1.209-0015

Nijimbere, C., Boulc'H, L., Haspekian, M. et Baron, G.-L. (2013, octobre). Apprendre l’informatique par la programmation des robots. Communication présentée à Sciences et technologies de l'information et de la communication (STIC) en milieu éducatif. Repéré à https://edutice.archives-ouvertes.fr/edutice-00875586/document

OECD. (2015). Schooling redesigned: Towards innovative learning systems. OECD. http://dx.doi.org/10.1787/9789264245914-en

Office québécois de la langue française (OQLF). (2001). Programmation. Dans Office québécois de la langue française (dir.), Grand dictionnaire terminologique. Repéré à http://gdt.oqlf.gouv.qc.ca/ficheOqlf.aspx?Id Fiche=2071728

Papert, S. (1981). Jaillissement de l'esprit scientifique : ordinateurs et apprentissage. Paris : Flammarion.

Robins, B., Amirabdollahian, F., Ji, Z. et Dautenhahn, K. (2010). Tactile interaction with a humanoid robot for children with autism: A case study analysis involving user requirements and results of an initial implementation. Dans 19th International Symposium in Robot and Human Interactive Communication (p. 704-711). http://dx.doi.org/10.1109/ROMAN.2010.5598641

Ruf, A., Mühling, A. et Hubwieser, P. (2014). Scratch vs. Karel: Impact on learning outcomes and motivation. Dans Proceedings of the $9^{\text {th }}$ Workshop in Primary and Secondary Computing Education (p. 50-59). New York, NY : ACM. http://dx.doi.org/10.1145/2670757.2670772

Sáez-López, J. M., Román-González, M., \& Vázquez-Cano, E. (2016). Visual programming languages integrated across the curriculum in elementary school: A two year case study using "Scratch" in five schools. Computers E Education, 97, 129-141. 
Shamsuddin, S., Yussof, H., Ismail, L. I., Mohamed, S., Hanapiah, F. A. et Zahari, N. I. (2012). Humanoid robot NAO interacting with autistic children of moderately impaired intelligence to augment communication skills. Procedia Engineering, 41, 1533-1538. http://dx.doi.org/10.1016/j.proeng.2012.07.346

Shiomi, M., Kanda, T., Howley, I., Hayashi, K. et Hagita, N. (2015). Can a social robot stimulate science curiosity in classrooms?. International Journal of Social Robotics, 7(5), 641-652. http://dx.doi.org/10.1007/s12369-015-0303-1

Smith, N., Sutcliffe, C. et Sandvik, L. (2014). Code club: Bringing programming to UK primary schools through Scratch. Dans J. D. Dougherty et K. Nagel (dir.), Proceedings of the 45 th ACM Technical Symposium on Computer Science Education (p. 517-522). New York, NY : ACM. http://dx.doi.org/10.1145/2538862.2538919

Toh, L. P. E., Causo, A., Tzuo, P.-W., Chen, I.-M. et Yeo, S. H. (2016). A review on the use of robots in education and young children. Journal of Educational Technology E' Society, 19(2), 148-163.

Trudel, L., Simard, C. et Vonarx, N. (2006). La recherche qualitative est-elle nécessairement exploratoire?. Recherches qualitatives, (hors série numéro 5), 38-45. Repéré à http://www.recherche-qualitative.qc.ca/documents/files/revue/hors serie/hors serie v5/trudel.pdf

Wing, J. M. (2006). Computational thinking. Communications of the ACM, 49(3), 33-35. http://dx.doi.org/10.1145/1118178.1118215

\section{Pour citer cet article}

Bugmann, J. et Karsenti, T. (2018). Apprendre à programmer un robot humanoïde : impacts sur des élèves de l'adaptation scolaire. Formation et profession, 26(1), 26-42. http://dx.doi.org/10.18162/fp.2018.460 